\title{
Learning to teach at a distance: exploring the role of electronic communication
}

\author{
Michelle Selinger \\ School of Education \\ The Open University, UK
}

\begin{abstract}
The Open University is now training the third cohort of PGCE students since its inception in 1994. Approximately 1,100 graduates are trained to teach in primary and secondary schools each year on an 18 month part-time distance taught course. In addition to traditional text based materials, video and audio cassettes, students are loaned an Apple computer for the duration of the course complete with Clarisworks, a Stylewriter printer, a modem and the electronic conferencing system, FirstClass. In this paper some of the issues that have emerged to date will be explored in an attempt to illustrate the potential of this form of communication for teacher education both in traditional institutions and in distance education for beginning teachers and teachers in the early years of their career. These include the establishment of the system, the training and workload implications for staff, and the students' perceptions based on questionnaire data gathered to date.
\end{abstract}

Keywords

Teacher education, communications, distance learning, open learning, teleconferencing

\section{THE COURSE STRUCTURE}

The computer conference structure in the Open University Postgraduate Certificate of Education (PGCE) course have been set up to mirror the course structure (see Selinger, 1996; Selinger, 1996a; Selinger and Parker, 1996 for more details). This means that students have the opportunity to participate on a voluntary basis in a range of different electronic groups. They are assigned to a tutor group of 15 students according to whether students are following the primary or secondary line of the course. These tutor groups are led by part-time tutors appointed to the Open University by a regional staff tutor in one of twelve Open University regions in England, Ireland and Wales. Each region has its own conference moderated by the 
staff tutor. There is also a 'lobby' for all students to contribute to discussion of a general nature and then there are subject conferences for each of the secondary subject lines and a primary conference. Primary students are actively encouraged to participate in the subject conference, thereby encouraging primary/secondary liaison (an atypical feature of a PGCE course). Technical queries are kept separate from the academic discussion in the 'ACS helpdesk'. There is also a 'General Chat' facility within the lobby which is for 'coffee time talk' and a group of students run a 'virtual pub' called 'Ye Olde Curiosity Shoppe' within General Chat.

Two questionnaires have been sent to the first cohort of students who finished the course in 1995. One was returned by about 700 students who completed the course and the other, a more detailed questionnaire, was returned by 300 students. A revised questionnaire will be sent to all second cohort students when they finish the course in July 1996. The results from the second cohort are expected to differ as these students have had more access to FirstClass. The differences have become particularly noticeable after 5 months on-line and are discussed later. The first cohort of students came on-line in February 1995, a year after starting the course. This was for a number of technical and administrative reasons. The second cohort came on-line six months into the course and the third cohort come on-line in April, just two months after the course started, and this is expected to become the norm for future presentations of the course.

\section{COMMUNICATION PROTOCOLS AND ISSUES}

There is no teaching built into the on-line conferences, but students are strongly encouraged to use the system. In the 1996 on-line presentation structure, students have access initially to tutorial and regional conferences in order to establish a pattern whereby the line of communication meets traditional Open University (OU) protocols, i.e. the tutor is the first point of contact. Having a system which permits ease of access to the course team has meant that the students are using the lobby as a forum for asking questions normally asked of tutors. One dilemma has arisen, which is currently in the process of being discussed and resolved; a single question to the meeting room can prevent the same question being asked in every tutorial group. However, if this happens and is responded to quickly, it soon becomes recognised as an efficient method for dealing with the questions, and the lobby becomes clogged up with queries instead of course-wide discussion and support; the purpose for which it was intended. The dilemma can be resolved by setting up separate sub-conferences in the lobby for course-related enquiries, or to delete any such messages and informing the 'offending' students via their private mailbox that the question must be forwarded to their tutor. There are advantages to the former. The course team are able to resolve any problems very quickly by posting a message to all the students and can then make adjustments to course materials for subsequent presentations of the course.

The ability to ask these questions, however, is important. In the evaluation questionnaire, it was very clear that seeking advice and clarifying instructions for things like the assessment portfolio was a very important factor in the students' use of FirstClass. It may also have contributed to their use of the system. Of the 266 students who responded to the more extended questionnaire and had said they had used 
FirstClass, 212 had used it 'for seeking information'. The next most utilised function was 'receiving moral support', with 127 students responding positively to that item.

\section{SUBJECT CONFERENCES}

Students were asked which conference had been the most valuable. Thirty six per cent reported the subject conferences as being the most useful. This was expected, and was one of the main reasons for setting up the FirstClass system. Secondary students only have access to one or two students following the same subject line in their tutor groups, as they are geographically allocated within either the primary or secondary line. These tutor groups meet together on five occasions throughout the course. Tutor groups in any one of the twelve regional areas of the Open University will also come together at Day Schools on another four occasions, with one seminar during the day allocated to subject issues. The FirstClass subject conferences therefore provide wider and greater access to all students following their line, and to the member of the course team and any tutors who are specialists in that area. Comments about the subject conferences were very positive:

"[I was] able to share information with like minded colleagues";

"We considered the practical teaching of maths and ideas for the classroom";

"Sharing school experience - good to see other students having similar difficulties and for sharing ideas";

"It gave me direct contact with the subject specialist on the course".

\section{BULLETIN BOARDS}

The bulletin boards, where messages from the course team are posted, and to which part-time tutors and students had read-only access, provide students with important information, changes and clarification of issues, as well as information about forthcoming events in their region or nationally. They also advise students about recent articles in newspapers or relevant TV and radio programmes. There are several bulletin boards: one general; one for each region; one for the primary conference; and one for each of the subject conferences. Many students reported only logging in to get information from the bulletin boards especially when they were in a hurry.

\section{$5 \quad$ STUDENTS' USE OF FIRST CLASS}

As stated earlier, the first cohort of students came on-line late in the course, therefore they did not all make full use of the system. Many reported this in their questionnaire responses. FirstClass was introduced almost a year after students had started their course. This was at a time when the last tutor-marked assignments were being written and the final school experience was nearing, so students were feeling very much under pressure. Another 'chore' was not welcomed by many. The reasons given for not 
making much use of the system were varied. Many gave lack of time (38 students) for the reasons given above, but others ranged from lack of confidence in the value of their contribution, to their lack of skill in using FirstClass or the computer.

"I only posted one message - I felt nervous about the system having never communicated in writing electronically' before";

"I lacked confidence in writing messages. I felt I had nothing to say";

"I never felt the need to";

"Lack of time. Feeling uncomfortable with computer apart from word processing".

The second cohort started to use FirstClass well before the second school experience and the use of it actually increased as school experience came round. The subject conferences became places to share resources and ideas while the lobby was to offer and gain moral support. This was also true of those in the first cohort of students who used the system. This is just one example:

"Thanks $\mathrm{S}$ for the brilliant idea for difficult classes. I tried your method almost verbatim on a horrendously "challenging" class. Only year 8, but all SEN pupils! Double period last thing Monday PM. Followed your system/gave them some "copying out" to do while I went round and assessed them for work which I thought would catch their (limited) imagination. I was delighted at the end of 100 minutes to feel a certain satisfaction. I recommend your tactics to others."

As the students go into their final school experience (referred to in the following messages as SE3), the lobby has been full of postings offering support and sharing concerns:

"I'm experiencing feelings of real terror. SE3 begins tomorrow. It's now a case of fight or flight. I don't fancy fisty-cuffs, so I might instead do a runner! This is crazy. I'm all grown up now! I should be able to handle this. I guess I'm afraid of failure......

Thanks for playing psychoanalyst, Lobby."

"I would just like to thank contributors to 'lobby' for the support that they provide. When I feel really low I read some of the messages and they always manage to cheer me up. It's reassuring to know that there are others who are going through exactly the same emotions. I know one thing, and that is, you're all special people and that's why you're doing what you're doing. Keep fighting and well done so far. I'm starting SE3 tomorrow as well and I'll go ahead knowing that I'm in good company!"

\section{ISSUES IN THE COURSE TEAM}

The course team, comprising of the PGCE director, 2 deputy directors, the subject and primary specialists and the regional staff tutors, also uses FirstClass to communicate. 
This has been particularly beneficial since regional staff tutors are located throughout the UK, therefore face-to-face meetings are expensive both in terms of time and cost, and there are a number of working groups to which they belong which have been set up to support and develop various parts of the programme. The structure of FirstClass enables separate conference areas to be set up, allowing members of each group to discuss issues between meetings, to set up agendas, to prepare joint working papers and to develop ideas. The general on-line area for the course team allows them to explore a range of issues, and there has been much wider opportunity to discuss and debate changes in policy before final decisions are made at course team meetings. The outcomes of use of FirstClass itself have been discussed by the course team. The regional staff tutors find the system valuable, in that contact can be easily made with students and tutors in their region, but they are unsure of how the protocols discussed earlier in this paper should be maintained. It has also increased their workload considerably, as one staff tutor pointed out. The process initiated a discussion that went on for several days, causing many to share their concerns about FirstClass as well as the benefits:

"At the moment I have this love/hate relationship with First Class as well! I love being able to get in touch with you all at the press of a button and feel secure in the fact that people in this conference know me to a greater or lesser degree and know my personality. As a result, consciously or sub-consciously, people will use that information in interpreting what I write.

On the other hand, turning on the machine and seeing a morass of red flags I find extremely stressful. I have to select what I am going to read, otherwise I will be here all night, but so many of those red flags are still there! I begin to feel inadequate! Is everybody else on the team reading all of this?

People can increase my work load at the press of a button and there are subtle inbuilt assumptions about the speed of reply. More stress!! My workload is increased as students can now reach me direct with the smallest of problems, by-passing their tutors and then feeling aggrieved if I re-direct them back to the tutor. Do I need this aggro and TIAS [Teaching in a second school] problems as well? No!

Although as many of you will know I am not a whiz kid on the computer, I have had a fair amount of experience as an advisory teacher of helping staff and pupils to get e-mail projects between schools and between different countries going. My learning curve is still going on. In terms of First Class, I may not always contribute to the conference, but I do get a lot out of reading the e-mail and will contribute when I have something to say. If I feel like this, I wonder how our less confident students feel!

While I agree that we need to encourage students to contribute, I don't think we should interpret the fact that reading rather than writing e-mails is somehow unsuccessful."

\section{TUTOR TRAINING}

Certainly the training issue for tutors and students has been reconsidered from time to time, as the benefits of electronic communication are accepted and considered to outweigh the problems. It is now deemed essential that tutors are sufficiently IT 
confident to use FirstClass, to support their students in coming on-line, and that any new staff members are fully trained through face-to-face, hands-on sessions. The student and tutor handbooks have been updated to offer more support, in an attempt to encourage students to make full use of the system, and tutors are given ideas about how they might encourage students to use the system. The acceptability of FirstClass by the course team, tutors and students alike has increased through usage. As one tutor reported:

"As a tutor I have been able to follow the fortunes of some of my past students, see how they're developing and generally have access to something that has never been available to me before. I think FirstClass should be extended to most if not all OU courses."

\section{ALUMNI}

What is referred to above is the alumni conference which has been set up for any student who has completed the course and has access to a modem and computer. Many of the students who have stayed on-line have purchased computers from their partner school if they did not want Apple machines. About 100 alumni initially expressed an interest in staying on-line, and a number of others have made contact in the past few months to inform us that they now have a computer and modem and want to join the alumni. Members of this conference were asked what they thought of FirstClass:

"I would say that using FirstClass as an alumni has been like an extension of the PGCE course. There is a constant supply of help and ideas as well as an exchange of views about every issue that faces any teacher not just students and newly qualified teachers. Most of my colleagues at school are only available to me for short times during the day, at lunch or break when they are not involved with other things; so learning from their experience is quite difficult as few have the time to spare and others have no inclination to let others benefit from their experience."

"I only have to log on and I can benefit from the best practice that is taking place in many schools and educational establishments around the country, not just my own; I can gain motivation and insight that other teachers cannot get."

Those who have accessed the system and utilised it fully are aware of the potential to communicate with other teachers and colleagues in the field of education. However, the benefits are not immediately apparent. All the partner schools from the first cohort of PGCE students were contacted and asked if they would like to be part of the alumni conferences. Only 100 schools responded positively, despite the fact they had access to a dedicated computer and modem which they gained as a result of having a student from the Open University. The service has been offered at no cost apart from telephone charges which are mostly at local call rate, yet still less than 50 teachers from these schools are active participants in FirstClass, let alone active contributors (a participant here is defined as someone who reads and/or contributes to messages). 


\section{SUMMARY}

The use of FirstClass has enabled students working at a distance to feel part of a community of Open University students. The debate and discussion that the system has allowed has meant that students have been able to share and express their fears and anxieties as well as their ideas and successes with a large community of others. The openness of this expression has been surprising and has been a factor in the success of the system.

Issues of training and support for staff use are being addressed, and the use of FirstClass is gradually being accepted as part of the normal workload. As more alumni come on-line each year, a growing community of teachers in schools all over the country will be able to communicate about their work, share ideas and resources, support each other through the traumatic early years of their careers, and become a new generation of teachers for whom computer mediated communications will become an expectation and a norm.

There is still much to be found about the ways in which teachers perceive the value of electronic networking. It is clear from the small survey reported here, that it is seen as a possibility by a very few. For others the costs and time are prohibitive when so many other factors challenge their daily routines and pressures. More detailed evaluation and follow-up interviews with cohort 2 students should provide more pieces of the jigsaw. This will enable the electronic community of teachers that could support and encourage long term professional development, from initial teacher education to continuing professional development, to be firmly established as a natural part of teachers' practice.

\section{REFERENCES}

Selinger, M. (1996) The role of electronic communication in supporting beginning teachers, in Proceedings of ED-MEDIA/ED-TELECOM 96, Boston, MA, June $17-22$.

Selinger, M. (1996a) Beginning teachers using IT: The Open University model. Journal for Information Technology in Teacher Education, April 1996.

Selinger, M. and Parker, B. (1996) Supporting teacher education through distance learning and electronic communications, in Proceedings of SITE 96 Conference, Phoenix, Arizona, March 1996.

\section{BIOGRAPHY}

Michelle Selinger is a lecturer in education at the Open University in the Centre of Research in Teacher Education. She writes distance learning material in the areas of IT across the curriculum and in mathematics education in pre-service teacher education and at masters level. She is a consultant to the UK National Council for Educational Technology. Her research focus is in the use of computer mediated communication as a medium for fostering a critical community of teachers. 